\title{
Conference and Dentistry Show announces new dates
}

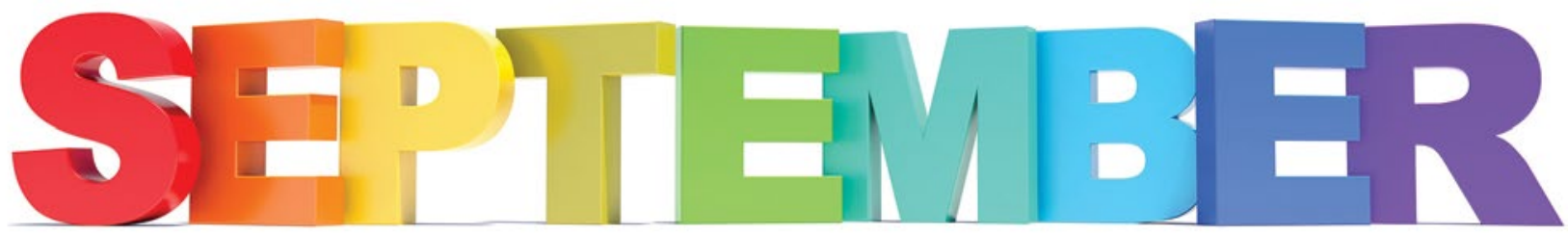

The British Dental Conference and Dentistry Show (BDCDS) will be postponed to Friday 11 and Saturday 12 September 2020 and will remain at the NEC Birmingham.

The original event was due to take place in May, but has been moved due to official advice regarding the current COVID-19 pandemic and the latest guidance on social distancing over the next 12 weeks [25 March].

This decision has been made in order to protect delegates, exhibitors, sponsors, partners, speakers and staff. The organisers hope the new date will enable the UK's dedicated dental professionals to focus on the current situation and come together as a united profession once the height of the COVID-19 crisis is over.

Alex Harden, Show Director of the British Dental Conference and Dentistry Show, said:
'We would like to thank everyone for their patience and understanding. While we have not taken this decision lightly, the health and safety of individuals involved is a top priority for us and we are committed to following all official guidance.

'We will be working hard to create another outstanding event in September, offering the plethora of first-class educational and networking opportunities the British Dental Conference and Dentistry Show has become known for. As such, CloserStill Media and the British Dental Association will be collaborating closely to ensure we present even more world-class speakers for delegates to hear from. We will also be liaising with partners, exhibitors and sponsors to ensure they have every opportunity to maximise on their time at the later event. If anyone has any questions, please do contact the CloserStill Media team.

'For now, I would encourage everyone to follow the guidance and stay safe during these difficult times.'

BDCDS is the UK's leading two-day exhibition and conference for dental professionals, providing clinical, leadership and practical training via an extensive educational programme and hands-on workshops. It is the UK's largest event of its kind, attracting over 9,000 unique delegates and over 400 exhibitors.

The British Dental Conference and Dentistry Show 2020 - 11 and 12 September - Hall 5, Birmingham NEC, co-located with DTS. Visit www.thedentistryshow.co.uk, call 02073485270 or email dentistry@ closerstillmedia.com.

\section{Faculty Dean calls for greater diversity}

The Dean of the Faculty of General Dental Practice (FGDP[UK]), Ian Mills, has called for the leaderships of dental organisations to better reflect the diversity of the profession.

In an address to Faculty members and professional leaders at the FGDP(UK) Annual Dinner and Awards on 12 March, he said dental leaders have a 'moral obligation' to ensure that their organisations 'support social justice through a culture and environment of inclusivity'.

He described as 'unacceptable' the persistence of 'many barriers which intimidate and inhibit certain individuals from engaging with dental organisations', and said that the best way to change perceptions and encourage engagement was by ensuring diversity of gender, age and ethnicity within boards at both national and local level.

Outlining the opportunity, he said diversity 'modulates attitudes and behaviours, undermines "group-think", and delivers improved performance within

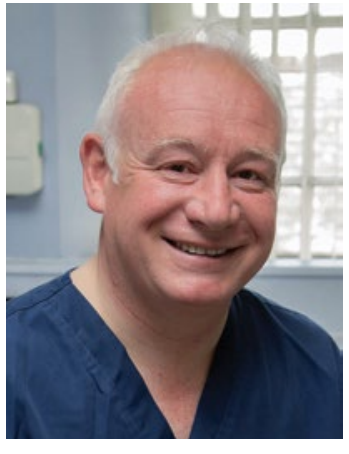

Ian Mills

an organisation'. He continued: 'Social and professional diversity reflects the diversity within our profession, and can provide insight into the different views, attitudes and beliefs which may exist. This is a healthy environment which can stimulate discussion, debate and reflection, and in turn, provides a degree of internal accountability. We need diversity to understand the changing needs of our profession, to connect with new members and ensure that we continue to be relevant'.
Giving the example of students and early careers dentists, he said that as Dean he 'can try to understand the challenges they face and what they need and want from the FGDP, but in reality, it is simply my interpretation. The different groups within dentistry need to have a voice'.

Dr Mills said this was 'particularly true of women in dentistry. He noted that when he was elected to the Faculty Board in 2003, there was only one female board member out of 30 (3.3\%), and that while this had improved to six out of 16 today (38\%), the board remained 'a male dominated environment' at a time when 'there are more female undergraduates than male, and the number of women on the dental register exceeds the number of men'.

He added that FGDP(UK) and the British Dental Association would be announcing details of a joint symposium on diversity in dental organisations, with a particular focus on gender balance, in due course. 